Research Article

\title{
The SLC Family Are Candidate Diagnostic and Prognostic Biomarkers in Clear Cell Renal Cell Carcinoma
}

\author{
Weiting Kang $\mathbb{D}^{1}{ }^{1}$ Meng Zhang $\mathbb{D}^{2},{ }^{2}$ Qiang Wang $\left(\mathbb{D},{ }^{3}\right.$ Da Gu $\mathbb{D}^{4},{ }^{4}$ Zhilong Huang $\mathbb{D}^{1,5}$ \\ Hanbo Wang $\mathbb{D}^{1}{ }^{1}$ Yuzhu Xiang $\mathbb{D}{ }^{1}{ }^{1}$ Qinghua Xia $\mathbb{D}^{1},{ }^{1}$ Zilian Cui $\mathbb{D}^{1},{ }^{1}$ and Xunbo Jin $\mathbb{D}^{1}$ \\ ${ }^{1}$ Department of Urology, Shandong Province Hospital Affiliated to Shandong University, 324 Jingwuweiqi Road, Jinan, \\ Shandong 250000, China \\ ${ }^{2}$ Department of General Surgery, The Second People's Hospital of Dongying, 28 Changchun Road, Dongying, \\ Shandong 257000, China \\ ${ }^{3}$ Department of Human Resources, Shandong Province Hospital Affiliated to Shandong University, 324 Jingwuweiqi Road, Jinan, \\ Shandong 250000, China \\ ${ }^{4}$ Department of Plastic Surgery, Jinan Central Hospital Affiliated to Shandong University, 105 Jiefang Road, Jinan, \\ Shandong 250000, China \\ ${ }^{5}$ Department of Urology, Lanling People's Hospital, 4 Jiankang Street, Lanling, Shandong 277700, China
}

Correspondence should be addressed to Zilian Cui; 122526195@qq.com and Xunbo Jin; 201720480@mail.sdu.edu.cn

Received 12 December 2019; Revised 29 February 2020; Accepted 24 March 2020; Published 2 May 2020

Academic Editor: Yu-Chang Tyan

Copyright (C) 2020 Weiting Kang et al. This is an open access article distributed under the Creative Commons Attribution License, which permits unrestricted use, distribution, and reproduction in any medium, provided the original work is properly cited.

Clear cell renal cell carcinoma (ccRCC) is the most common lethal subtype of renal cancer, and changes in tumor metabolism play a key role in its development. Solute carriers (SLCs) are important in the transport of small molecules in humans, and defects in SLC transporters can lead to serious diseases. The expression patterns and prognostic values of SLC family transporters in the development of ccRCC are still unclear. The current study analyzed the expression levels of SLC family members and their correlation with prognosis in ccRCC patients with data from Oncomine, Gene Expression Profiling Interactive Analysis (GEPIA), The Cancer Genome Atlas (TCGA), cBioPortal, the Human Protein Atlas (HPA), the International Cancer Genome Consortium (ICGC), and the Gene Expression Omnibus (GEO). We found that the mRNA expression levels of SLC22A6, SLC22A7, SLC22A13, SLC25A4, SLC34A1, and SLC44A4 were significantly lower in ccRCC tissues than in normal tissues and the protein expression levels of SLC22A6, SLC22A7, SLC22A13, and SLC34A1 were also significantly lower. Except for SLC22A7, the expression levels of SLC22A6, SLC22A13, SLC25A4, SLC34A1, and SLC44A4 were correlated with the clinical stage of ccRCC patients. The lower the expression levels of SLC22A6, SLC22A13, SLC25A4, SLC34A1, and SLC44A4 were, the later the clinical stage of ccRCC patients was. Further experiments revealed that the expression levels of SLC22A6, SLC22A7, SLC22A13, SLC25A4, SLC34A1, and SLC44A4 were significantly associated with overall survival (OS) and disease-free survival (DFS) in ccRCC patients. High SLC22A6, SLC22A7, SLC22A13, SLC25A4, SLC34A1, and SLC44A4 expression predicted improved OS and DFS. Finally, GSE53757 and ICGC were used to revalidate the differential expression and clinical prognostic value. This study suggests that SLC22A6, SLC22A7, SLC22A13, SLC25A4, SLC34A1, and SLC44A4 may be potential targets for the clinical diagnosis, prognosis, and treatment of ccRCC patients.

\section{Introduction}

Kidney cancer is one of the most common malignant tumors in the urinary system, and more than half of patients are found by chance and have no clinical symptoms. In 2019, the number of new cases of kidney cancer in the United
States was 73,820 , ranking fifth in the number of new cases in men and eighth in the number of new cases in women [1]. Renal cell carcinoma (RCC) is the most common form of kidney cancer and accounts for $90 \%$ of all tumors, with ccRCC being the most common histology $(75 \%)[2,3]$. ccRCC is derived from the proximal tubular epithelium of 
the kidney and exhibits unique histological characteristics. Macroscopically, ccRCC are golden yellow in section and often have bleeding, necrosis, and cystic areas. Histologically, ccRCC usually consists of tumor cells with clear cytoplasm, surrounded by nests or tubules with a rich network of blood vessels. The clear appearance of the cytoplasm is due to the accumulation of glycogen and lipids [4]. Different renal cancer cell subtypes have different genetic changes and different prognoses. The cure rate is high for patients with early, localized disease, with 5-year survival at more than 90\% [5]. In contrast, 5-year survival drops to $12 \%$ for patients with distant metastatic disease.

Epigenetic changes in ccRCC may be related to its prognosis and treatment. Genetic markers of ccRCC include biallelic inactivation of Von Hippel-Lindau (VHL) tumor suppressor genes; negative regulators of hypoxia-inducible factor (HIF) protein; copy number changes of chromosome $3 \mathrm{p}, 5 \mathrm{q}$, and $14 \mathrm{q}$ genes; and chromatin-modifying enzyme high mutation frequencies, such as protein polybromo-1 (PBRM1), SET domain containing 2 (SETD2), and BRCA1associated protein-1 (BAP1) [6]. Currently, multitarget tyrosine kinase inhibitors (TKIs) and mechanistic target of rapamycin kinase (mTOR) inhibitors have become major breakthroughs in the treatment of ccRCC. In addition, immune checkpoint inhibitors have also become effective treatment options against advanced ccRCC. However, ccRCC has been called "metabolic disease," and its multiple bioenergy pathways are changed [7,8]. Extensive metabolic reprogramming in glucose, lipid, and amino acid metabolism has largely promoted the clear cell phenotype [9], but the genetic mechanism of these changes is not fully understood.

In recent years, the role of membrane transporters in cancer has received increasing attention. Two superfamily transporters have been discovered, namely, the ATPbinding cassette (ABC) family and SLC family. SLC transporters help ingest certain essential molecules [10], such as amino acids and glucose, and regulate metal absorption, mediating the normal functions of important enzymes [11]. Previous studies have found several essential nutrient transporters, such as the glucose transporter SLC2A1 [12] and the amino acid transporters SLC1A5, SLC7A5, SLC6A14, SLC7A11, and SLC38A2 [13-17], which are upregulated in cancer as tumor promoters. SLC39A1 regulates the malignant potential of prostate cancer cells by inhibiting the nuclear factor kappa B (NF- $\kappa \mathrm{B})$ signaling pathway, and SLC39A1 may play a role as a tumor suppressor gene in prostate cancer [18]. A good understanding of the differential expression of SLC transporters in various cancer cells can provide a theoretical basis for the development of new strategies for the treatment of cancer.

Although some scholars have studied the differential expression of SLC mRNA in ccRCC, it was found that SLC10A2 was significantly downregulated in TKI-treated samples and SLC10A2 was upregulated in ccRCC compared with adjacent kidney tissues in paired The Cancer Genome Atlas (TCGA) samples. High SLC10A2 expression was associated with good prognosis of ccRCC [19]. The results were contradictory. In this study, a bioinformatics analysis was performed to explore the roles of multiple SLCs in ccRCC. We analyzed the expression and mutation of different SLCs in ccRCC patients to determine their expression patterns, potential functions, and prognostic values in ccRCC.

\section{Materials and Methods}

2.1. Screening Cancer-Associated Genes in ccRCC and the Expression Level of the SLC Family Genes in Pancancer Using Oncomine Datasets. The Oncomine datasets (http:// www.oncomine.org) integrate RNA and DNA sequencing data from sources such as the Gene Expression Omnibus (GEO), TCGA, and published literature. We used the Jones Renal dataset to screen overexpressed and underexpressed genes in the associated concept tab of Oncomine. Compared with normal tissues, we screened top $10 \%$ overexpressed and $10 \%$ underexpressed genes in Jones Renal dataset of ccRCC. These screened genes were used in the next step of data analysis.

We use Oncomine to analyze the expression levels of the SLC family genes in pancancer (cancer vs. normal). The $P$ value was defined as 0.01 , fold change (FC) was defined as 2, gene rank was defined as all, and data type was also defined as all.

2.2. The Differential Expression and Prognostic Value of the SLC Family Genes Using Gene Expression Profiling Interactive Analysis (GEPIA) Dataset. GEPIA (http:/gepia .cancer-pku.cn/) is a newly developed interactive web server for analyzing the RNA sequencing expression data of 9,736 tumor samples and 8,587 normal samples from TCGA and Genotype-Tissue Expression (GTEx) projects using a standard processing pipeline [20]. We used GEPIA to analyze the differential expression of the SLC family in ccRCC and its relationship with clinical stage. TCGA normal tissue and GTEx normal tissue were matched. The $P$ value and fold change were defined as 0.01 and 2, respectively. In addition, the GEPIA was used to analyze the correlation between the SLC family and the prognosis of ccRCC. The group cutoff for the survival analysis was $50 \%$, and the $P$ value and fold change were defined as 0.01 and 2 , respectively.

2.3. Analysis of Genetic Alteration in the SLC Family Genes Using cBioPortal. TCGA (http://www.cancer.gov/tcga) collected, characterized, and analyzed cancer samples from over 11,000 patients over a 12-year period. TCGA data, resources, and materials were originally published by the National Cancer Institute. We used the cBioPortal tool to analyze 538 cases of kidney renal clear cell carcinoma (TCGA, Firehose Legacy). The cBioPortal for cancer genomics (http://www.cbioportal.org/) provides the visualization, analysis, and download of large-scale cancer genomic datasets [21, 22]. The genomic profiles included mutations, putative copy number alterations from the Genomic Identification of Significant Targets in Cancer (GISTIC), mRNA expression $Z$-scores (RNASeq V2 RSEM), and protein expression $Z$-scores (RPPA). In addition, the correlation between the expression levels of the SLC family genes was analyzed using a plot module of cBioPortal. Data type was 
selected mRNA and the mRNA profile was selected mRNA expression $Z$-scores (RNASeq V2 RSEM). Then, we used the bioconductor "pheadmap" package for drawing.

\subsection{Detection of the SLC Family Protein Expression in} Tissues by the Human Protein Atlas (HPA). The HPA was a Swedish-based programme initiated in 2003 with the aim of mapping all the human proteins in cells, tissues, and organs using the integration of various -omics technologies, including antibody-based imaging, mass spectrometry-based proteomics, transcriptomics, and systems biology. Immunohistochemistry (IHC) images of ccRCC were kindly provided by the Protein Atlas Project, which is publicly available (http://www.proteinatlas.org). In the Human Protein Atlas, all images of tissues stained by immunohistochemistry were manually annotated by a specialist followed by verification by a second specialist. Staining intensity was divided into four levels: negative, weak, medium, and strong. According to the fraction of stained cells, staining quantity was also divided into four levels: none, $<25 \%, 25-75 \%$, and $>75 \%$. Protein expression levels were based on staining intensity and staining quantity. The classification criteria for protein expression levels were as follows: negative, not detected; weak and $<25 \%$, not detected; weak combined with either $25-75 \%$ or $75 \%$, low; moderate and $<25 \%$, low; moderate combined with either $25-75 \%$ or $75 \%$, medium; strong and $<25 \%$, medium; and strong combined with either $25-$ $75 \%$ or $75 \%$, high.

All slides with SLC22A6, SLC22A7, SLC22A13, SLC25A4, SLC34A1, and SLC44A4-staining were downloaded from the HPA. The average number of normal tissues was 5 , and the average number of cancer tissue was 36.8 . The protein expression levels in cancer and normal tissues were compared in terms of staining, intensity, and quantity. The data was subjected to a Mann-Whitney $U$ test using SPSS to calculate the $P$ value. When performing statistical calculations, the weight of each patient was 1 . If a patient had multiple samples, 1 was equally divided into each sample. The $P$ value was defined as 0.01 .

2.5. The Protein-Protein Interaction (PPI) Network Construction and Analysis of Modules. The STRING database (http://http://string-db.org/) provides a significant association of protein-protein interactions [23]. Cytoscape is used for the visual exploration of interaction networks [24]. In this study, PPI networks were analyzed by the STRING database and subsequently visualized by using Cytoscape. The minimum required interaction scores were defined as high confidence (0.700). The max number of interactors to show was set as 100 . Cytoscape was then used to visualize the PPI network. The Cytoscape plugin Molecular Complex Detection (MCODE) was used to screen out modules of PPI networks, and the degree cutoff $=2$, node score cutoff $=0.2, k-$ score $=2$, and $\max$ depth $=100$ [25].

2.6. Metascape for Functional Enrichment and Kyoto Encyclopedia of Genes and Genomes (KEGG) Analysis. The aim of Metascape (http://metascape.org/gp/index.html) is to develop a set of reliable, productive, and intuitive tools that help a biomedical research community to analyze gene/protein lists and make better data-driven decisions. Metascape combines feature enrichment, cross analysis, gene annotation, and other search functions, including more than 40 independent knowledge bases [26]. We used Metascape to perform functional enrichment and KEGG analysis of SLC family member protein and its interacting proteins. Only terms with $P<0.01$, a minimum count of 3 , and an enrichment factor $>1.5$ were considered significant.

\subsection{Verify the Differential Expression of the SLC Family Genes} Using GSE53757 Dataset. The data of ccRCC were derived from the GEO. We used the Bioconductor "GEOquery" package to download the GSE53757 dataset from the GEO. Subsequently, differential expression analysis was performed using the Bioconductor "limma" package. The $P$ value and fold change were defined as 0.01 and 2, respectively. Then, the bioconductor "pheatmap" package was used to draw the heat map and the bioconductor "ggplot" package was used to draw the boxplot.

2.8. Validate the Prognostic Value of the SLC Family Genes Again Using the International Cancer Genome Consortium Dataset. The package "UCSCXenaTools" of R software was used to download the expression data and clinical data of ccRCC from the ICGC. The downloaded dataset included a total of 594 specimens, of which 72 were normal tissues and 30 patients had a survival time of less than 30 days. A total of 492 ccRCC patients were included for survival analysis with the $\mathrm{R}$ package "survival." The $P$ value was defined as 0.01 .

\section{Results}

3.1. Screening the SLC Family in ccRCC. First, Oncomine was used to select ccRCC in the Jones Renal database, which includes 23 pairs of cancer and normal tissues. The top $10 \%$ overexpressed genes and 10\% underexpressed genes, totalling 2,524 genes, were selected, of which 50 were SLC family genes. Second, the SLC family genes with the differential expression in ccRCC were screened again using GEPIA. The $P$ value and fold change were defined as 0.01 and 2, respectively. Third, GEPIA was used to screen SLC family genes associated with OS and DFS in ccRCC. The group cutoff for the survival analysis was 50\%, and the $P$ value and fold change were defined as 0.01 and 2 , respectively. The specific process is shown in Figure 1. Finally, six SLC family genes were screened: SLC22A6, SLC22A7, SLC22A13, SLC25A4, SLC34A1, and SLC44A4.

3.2. The Expression Levels of SLC Family Genes in Pancancer. We used the Oncomine database to analyze the expression levels of SLC22A6, SLC22A7, SLC22A13, SLC25A4, SLC34A1, and SLC44A4 in various cancers and their normal tissues (Figure 2). Among the various cancers examined, SLC22A6, SLC22A7, SLC22A13, SLC25A4, and SLC34A1 were significantly downregulated, while SLC44A4 showed different expression patterns in different cancers (upregulated or downregulated). In kidney cancer, SLC22A6, 


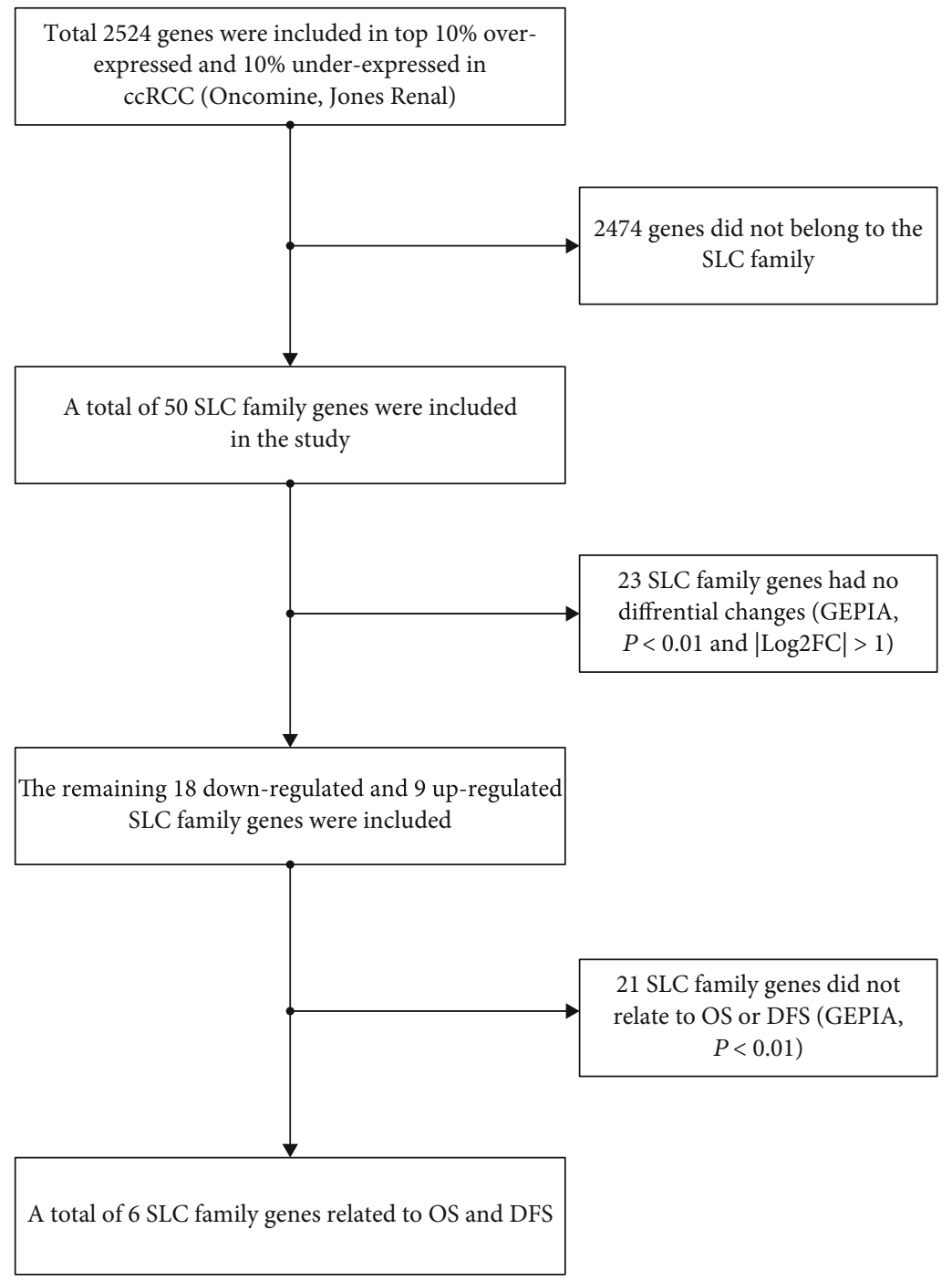

FIGURE 1: Screening process of the SLC family. OS: overall survival; DFS: disease-free survival.

SLC22A7, SLC22A13, SLC25A4, SLC34A1, and SLC44A4 were significantly downregulated.

3.3. The Difference Analysis of SLC Family Genes and Its Relationship with Clinical Stage. The expression levels of SLC22A6, SLC22A7, SLC22A13, SLC25A4, SLC34A1, and SLC44A4 in ccRCC were compared using the GEPIA database. A total of $523 \mathrm{ccRCC}$ cancer tissues and 100 normal tissues were included. As shown in Figures 3(a), the expression levels of SLC22A6, SLC22A7, SLC22A13, SLC25A4, SLC34A1, and SLC44A4 mRNAs in ccRCC were significantly decreased, and the difference was statistically significant $\left(P<0.01\right.$ and $\left.\left|\log _{2} \mathrm{FC}\right|>1\right)$.

Further studies using the GEPIA database revealed that the expression levels of SLC22A6, SLC22A7, SLC22A13, SLC25A4, SLC34A1, and SLC44A4 in ccRCC correlated with the clinical stage of the cancer, as shown in Figure 3(b). The expression levels of SLC22A6, SLC22A13, SLC25A4, SLC34A1, and SLC44A4 were significantly correlated with the clinical stage of the cancer. The lower the expression levels of SLC22A6, SLC22A13, SLC25A4, SLC34A1, and
SLC44A4 were, the later the clinical stage of the ccRCC patients was. There was no correlation between the expression level of SLC22A7 and the clinical stage of the cancer.

3.4. The Differential Expression of SLC Family Proteins Was Detected by Immunohistochemistry. The expression levels of SLC22A6, SLC22A7, SLC22A13, SLC25A4, SLC34A1, and SLC44A4 proteins in ccRCC were studied using the HPA. We downloaded all slides stained with SLC22A6, SLC22A7, SLC22A13, SLC25A4, SLC34A1, and SLC4A4 from the HPA. A total of 30 normal tissues were included, and the average number of normal tissues per gene was 5 . A total of 221 cancer tissues were included, and the average number of cancer tissues per gene was 36.8. The protein expression levels in cancer and normal tissues were compared in terms of staining, intensity, and quantity. Compared with normal tissues (as shown in Figure 4 and Table 1), the expression levels of SLC22A6, SLC22A13, and SLC34A1 proteins in ccRCC tissues were significantly decreased in terms of staining, intensity, and quantity. Compared with normal tissues, the expression levels of SLC22A7 proteins in ccRCC tissues 


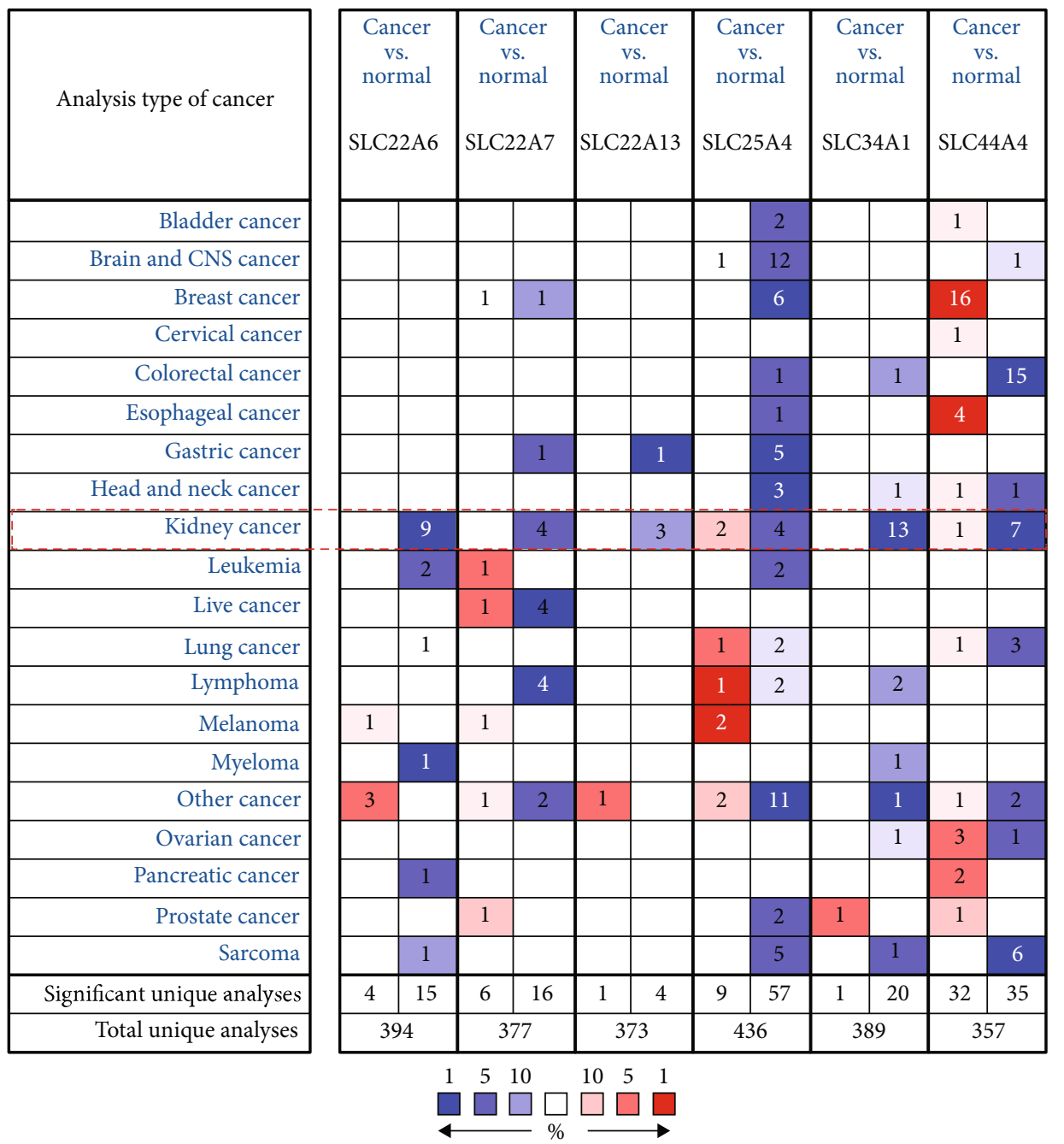

Figure 2: The expression levels of SLC family genes in pancancer (cancer vs. normal). The degree of cell color was determined by the best gene rank percentile. Red represented overexpression and blue represented underexpression. The number in the cell indicates the number of datasets that reached the threshold in the corresponding datasets of cancers.

were significantly decreased in terms of staining and quantity. In cancer tissues, SLC25A4 and SLC44A4 proteins did not change significantly in terms of staining, intensity, and quantity.

3.5. Association of SLC Family Genes with the Prognosis of Patients with ccRCC. The impact of SLC family genes on the survival of ccRCC patients was analyzed with the GEPIA database. As shown in Figure 5, the expression levels of SLC22A6, SLC22A7, SLC22A13, SLC25A4, SLC34A1, and SLC44A4 in ccRCC were significantly correlated with the OS and DFS of patients, and the difference was statistically significant $(P<0.01)$. High SLC22A6, SLC22A7, SLC22A13, SLC25A4, SLC34A1, and SLC44A4 expression predicted improved OS and DFS.

3.6. The Genetic Alterations of SLC Family Genes in ccRCC. cBioPortal was used to analyze the genetic alterations and correlations of SLC family genes in ccRCC. As shown in Figures 6, genetic alterations in SLC22A6, SLC22A7,
SLC22A13, SLC25A4, SLC34A1, and SLC44A4 occurred in $42.2 \%$ of ccRCC patients, with SLC22A6 in 3\%, SLC22A7 in $2.2 \%$, SLC22A13 in $12 \%$, SLC25A4 in $3 \%$, SLC34A1 in $18 \%$, and SLC44A4 in 4\%. SLC22A6, SLC22A7, SLC25A4, and SLC44A4 were mainly altered by mRNA high, SLC22A13 was mainly altered by a deep deletion, and SLC34A1 was mainly altered by an amplification.

3.7. The Correlation between Different SLC Family Genes and Construction of PPI Network. We determined the correlations between SLC family genes by analyzing their mRNA expression levels (RNASeq V2 RSEM) via the cBioPortal online tool for ccRCC (TCGA, Provisional), and Pearson's correlation was included. The results indicated positive correlations between the following: SLC22A6 with SLC22A13 and SLC34A1; SLC22A7 with SLC22A13 and SLC34A1; and SLC22A13 with SLC34A1 (Figure 7(a)). In particular, there were significant positive correlations between SLC22A6 and SLC22A13 and SLC22A13 and SLC34A1. 


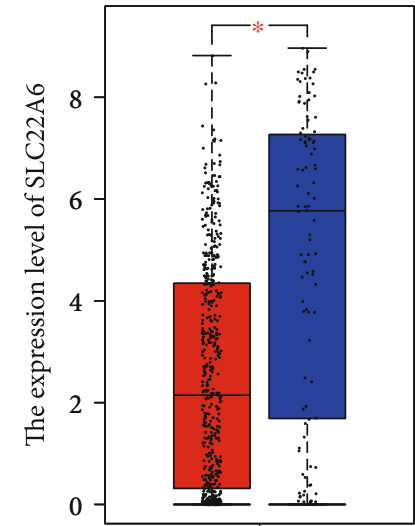

Cancer Normal

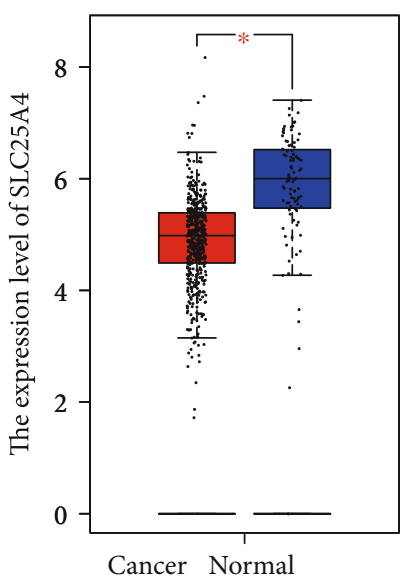

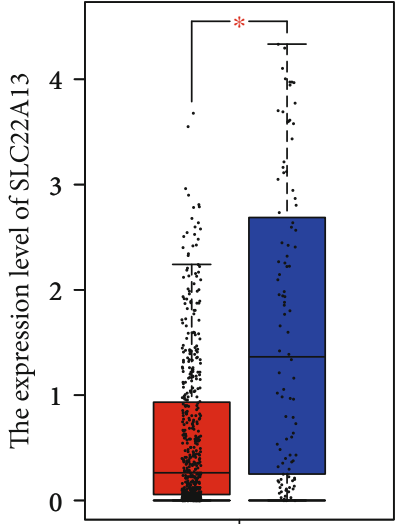

Cancer Normal

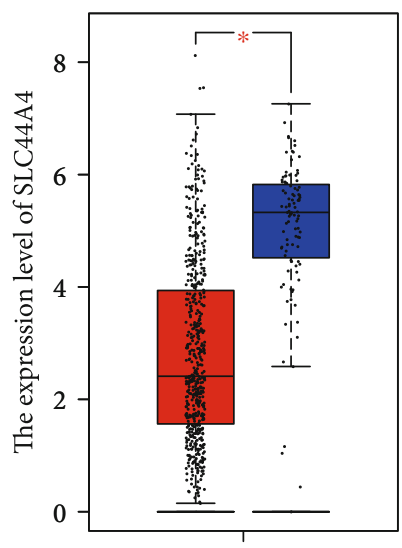

Cancer Normal

(a)
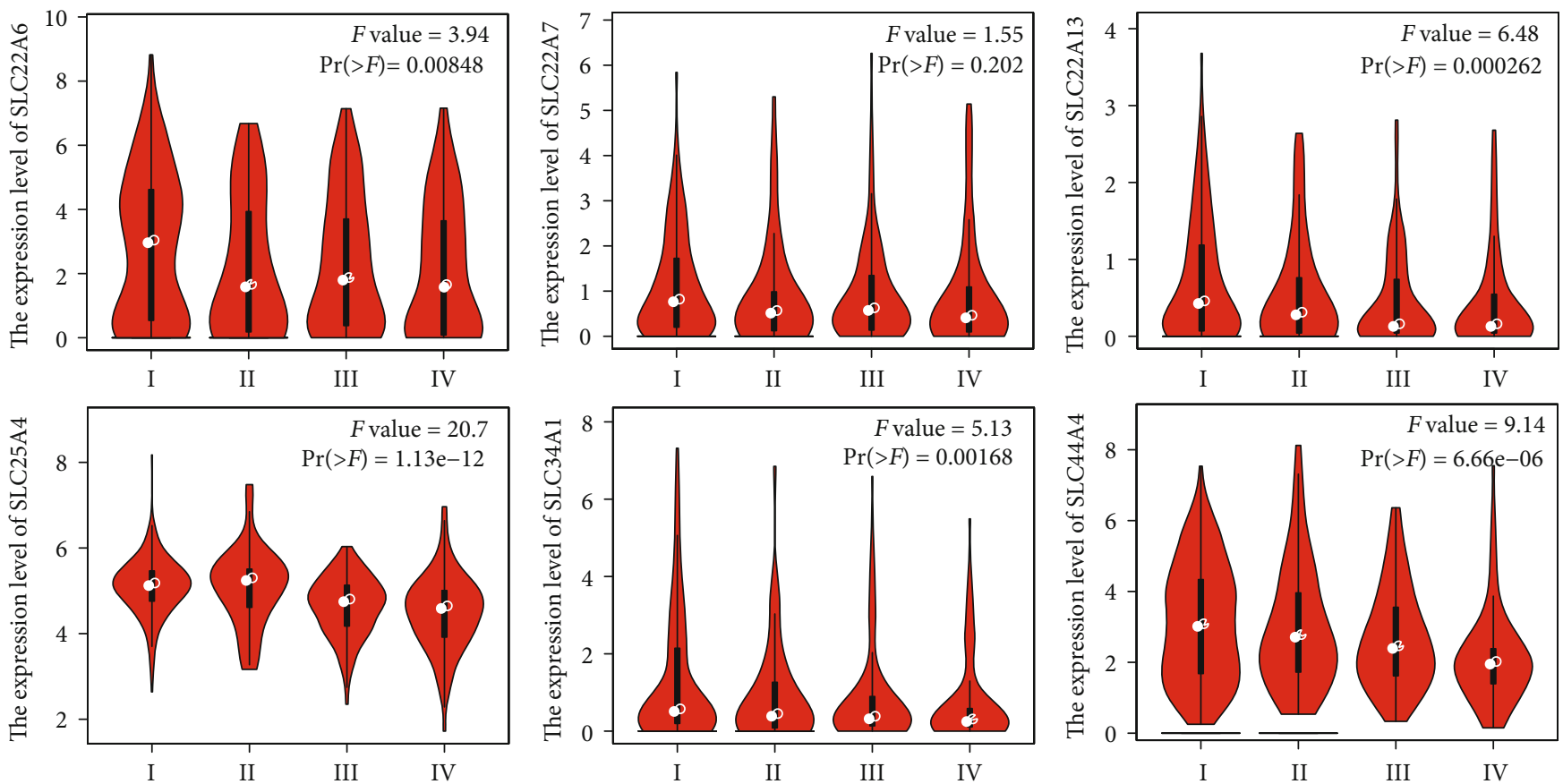

(b)

FIGURE 3: The expression level of SLC family genes and its correlation with tumor stage in ccRCC (GEPIA). (a) The boxplot of the SLC family gene expression level. ${ }^{*} P<0.01$ and $|\log 2 \mathrm{FC}|>1$. (b) The correlation between the expression of SLC family genes and tumor stage (I, II, III, and IV) in ccRCC. $\operatorname{Pr}(>\mathrm{F})<0.01$ meant the difference was statistically significant. 

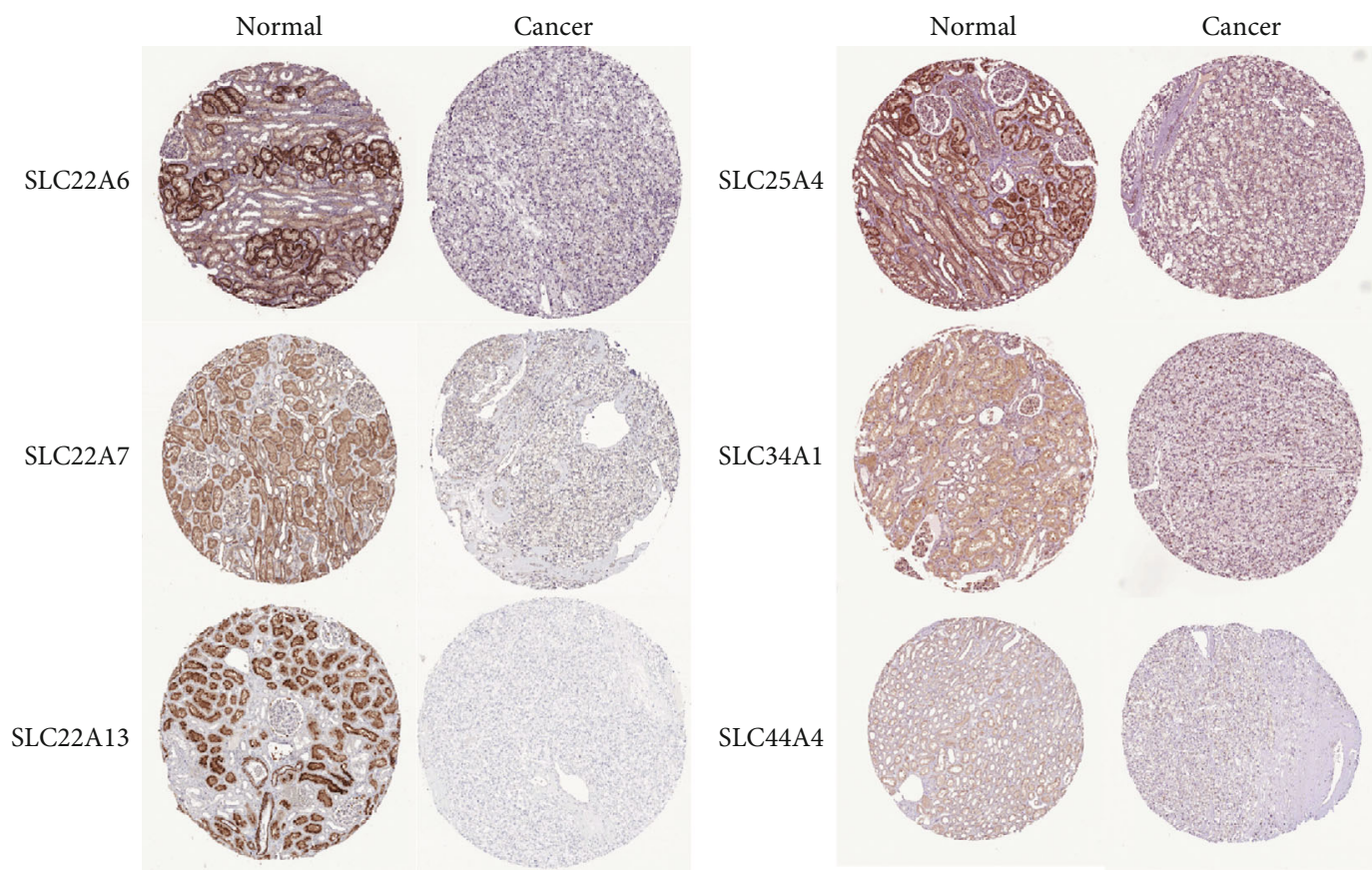

Figure 4: Representative tissue microarray (TMA) slides with SLC22A6, SLC22A7, SLC22A13, SLC25A4, SLC34A1, and SLC44A4 staining. All slides were retrieved from the Human Protein Atlas (HPA, http://www.proteinatlas.org). Magnification, $\times 100$.

Then, the SLC family genes were used to construct the PPI network by STRING. When building a PPI network, high confidence equals 0.700 , and the max number of interactors to show was 100. After the PPI network was constructed, the network statistics are as follows: the number of nodes was 106, number of edges was 1053, average node degree was 19.9, avg. local clustering coefficient was 0.771 , expected number of edges was 121, and PPI enrichment $P$ value was $1.0 e-16$. Then, we use the Cytoscape tool to visualize the PPI network (Figure 7(b)). Finally, the Molecular Complex Detection (MCODE) plugins of Cytoscape were utilized to choose a hub cluster of the PPI network. The hub cluster contained 36 nodes and 561 edges. The genes concentrated in the hub cluster, such as translocase of inner mitochondrial membrane (TIMM) family genes, translocase of outer mitochondrial membrane (TOMM) family genes, and ATP family genes, were mainly involved in protein targeting to mitochondrion, mitochondrial transport, etc.

3.8. Functional Enrichment and KEGG Analysis of SLC Family Genes and Their Interacting Genes. The functions of the SLC family genes and the genes significantly associated with SLC family gene alterations were predicted by GO and KEGG analyses with Metascape (Figure 8). The GO enrichment analysis predicted the functional roles of the target host genes on the basis of three aspects: biological process, cellular component, and molecular function. The biological process terms mainly included GO: 0007005 (mitochondrion organization), GO: 0006820 (anion transport), GO: 0007006 (mitochondrial membrane organization), GO: 0015672 (monovalent inorganic cation transport), and GO: 0015893 (drug transport); the cellular component terms mainly included GO: 0005740 (mitochondrial envelope), GO: 0005758 (mitochondrial intermembrane space), GO: 0005744 (TIM23 mitochondrial import inner membrane translocase complex), GO: 0005759 (mitochondrial matrix), and GO: 0005742 (mitochondrial outer membrane translocase complex); and the molecular function terms mainly included GO: 0022804 (active transmembrane transporter activity), GO: 0008509 (anion transmembrane transporter activity), GO: 1904680 (peptide transmembrane transporter activity), GO: 0015238 (drug transmembrane transporter activity), and GO: 0015288 (porin activity).

The KEGG can define the pathways related to the functions of the SLC family gene and their interacting genes (Figure 8). The relevant signaling pathways were hsa05012 (Parkinson's disease), M00009 (citrate cycle (TCA cycle, Krebs cycle)), hsa00190 (oxidative phosphorylation), and hsa04976 (bile secretion).

3.9. Validation of the Differential Expression of SLC Family Genes in ccRCC Using GSE53757 Datasets. We downloaded the GSE53757 dataset from GEO. It contained 144 tissues, of which 72 are normal tissues and 72 are cancer tissues. Cancer tissue includes 24 cancer tissues in clinical stage 1,19 cancer tissues in clinical stage 2, 14 cancer tissues in clinical stage 3 , and 15 cancer tissues in clinical stage 4. After the difference analysis using the "limma" package, we found that the expression levels of SLC22A6, SLC22A7, SLC22A13, SLC25A4, SLC34A1, and SLC44A4 in ccRCC were significantly decreased $(P<0.01$, as shown in Figures 9(a) and $9(\mathrm{~b})$ ), and the difference was statistically significant. Except for SLC25A4, the abundance difference of SLC22A6, SLC22A7, SLC22A13, SLC34A1, and SLC44A4 was more than 2 -fold. 


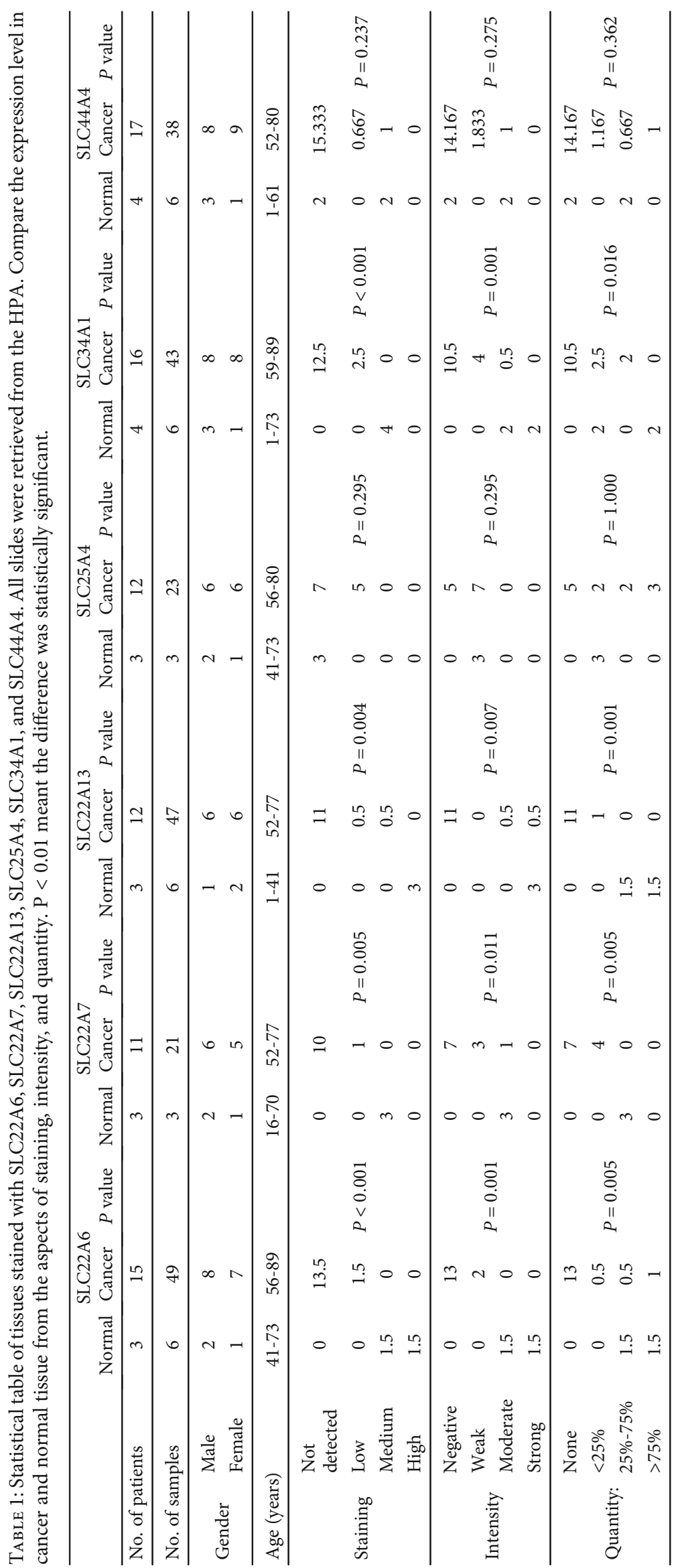



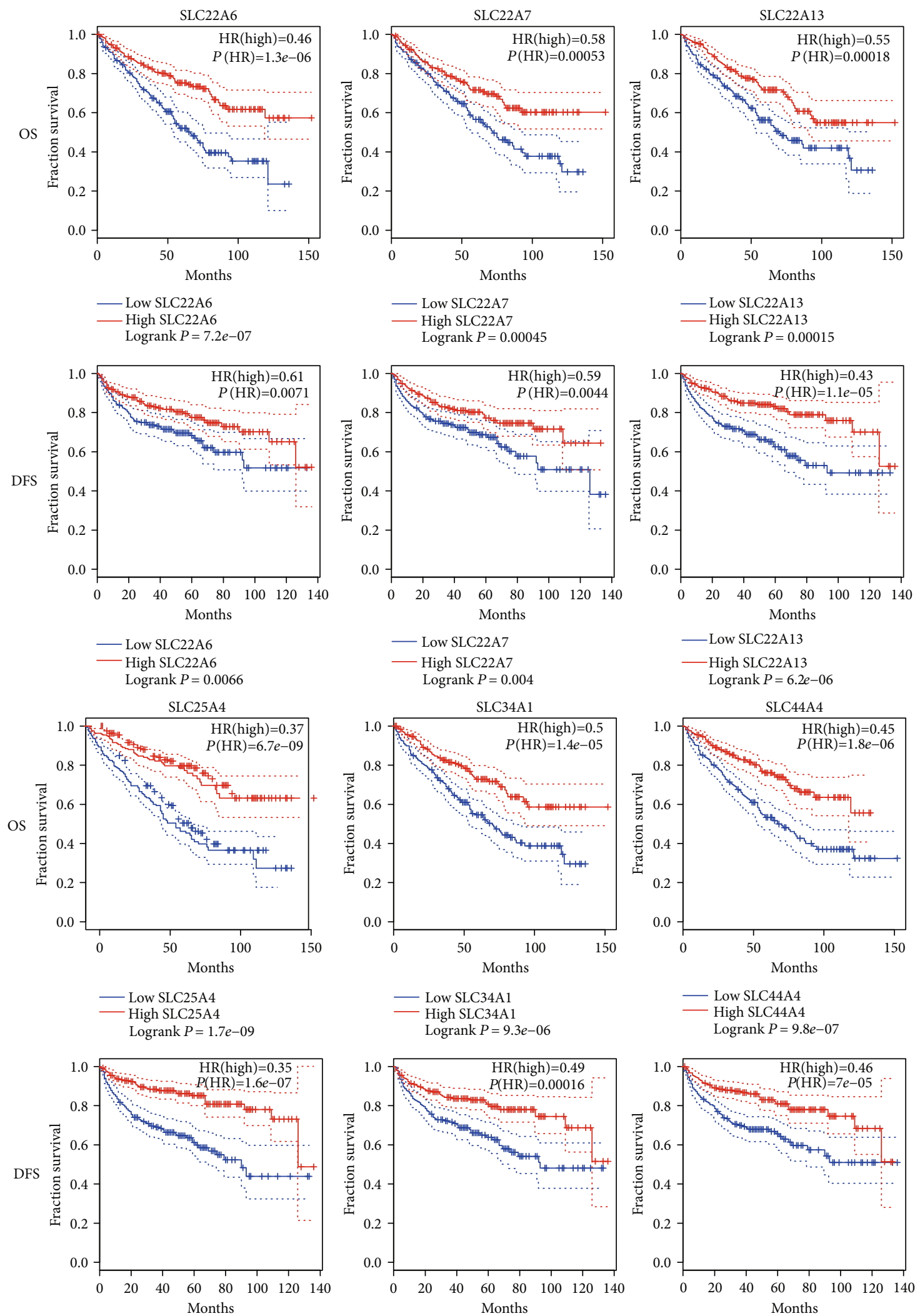

$$
\begin{aligned}
& - \text { Low SLC34A1 } \\
& \text { High SLC34A1 } \\
& \text { Logrank } P=9.3 e-06
\end{aligned}
$$

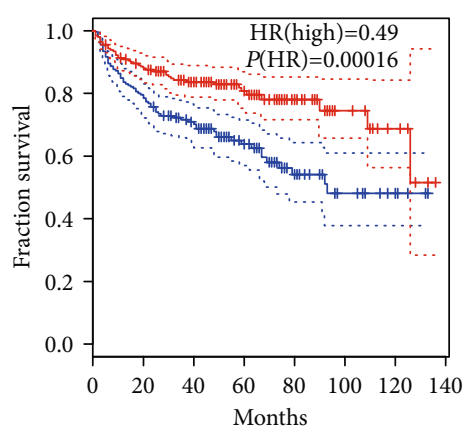

$$
\begin{aligned}
& - \text { Low SLC44A4 } \\
& \text { High SLC44A4 } \\
& \text { Logrank } P=9.8 e-07
\end{aligned}
$$
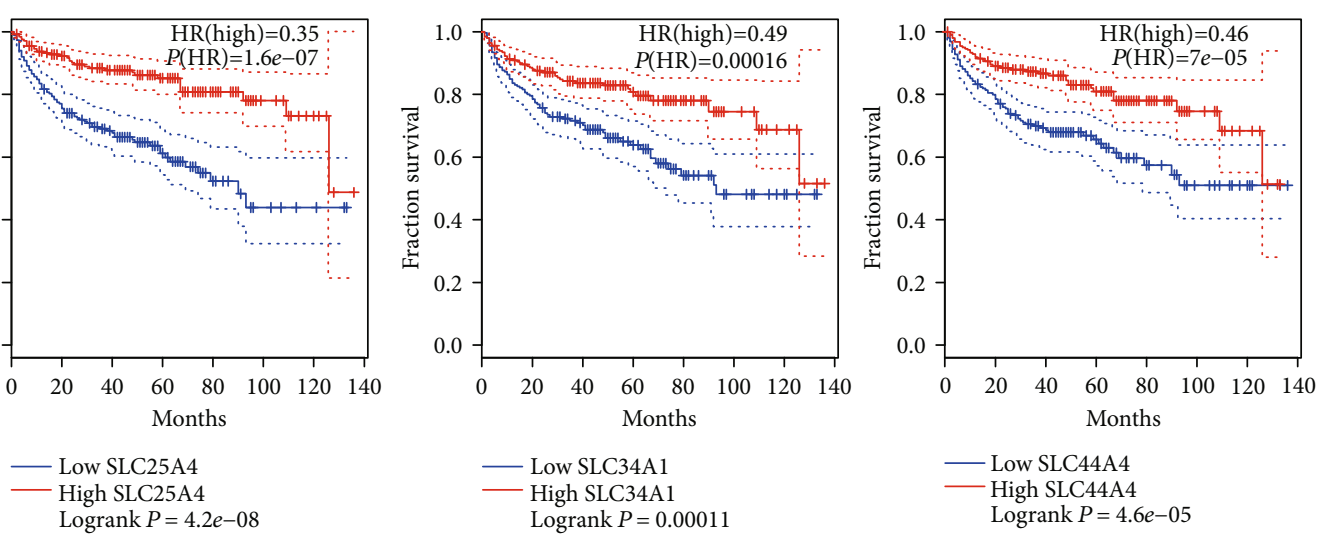

$$
\begin{aligned}
& \text { Low SLC44A4 } \\
& \hline \text { High SLC44A4 } \\
& \text { Logrank } P=4.6 e-05
\end{aligned}
$$

FIGURE 5: Kaplan-Meier survival curves for OS and DFS according to the expression level of SLC family genes in ccRCC (GEPIA). OS: overall survival; DFS: disease-free survival; HR: hazard ratio. Logrank $P<0.01$ meant the difference was statistically significant. 


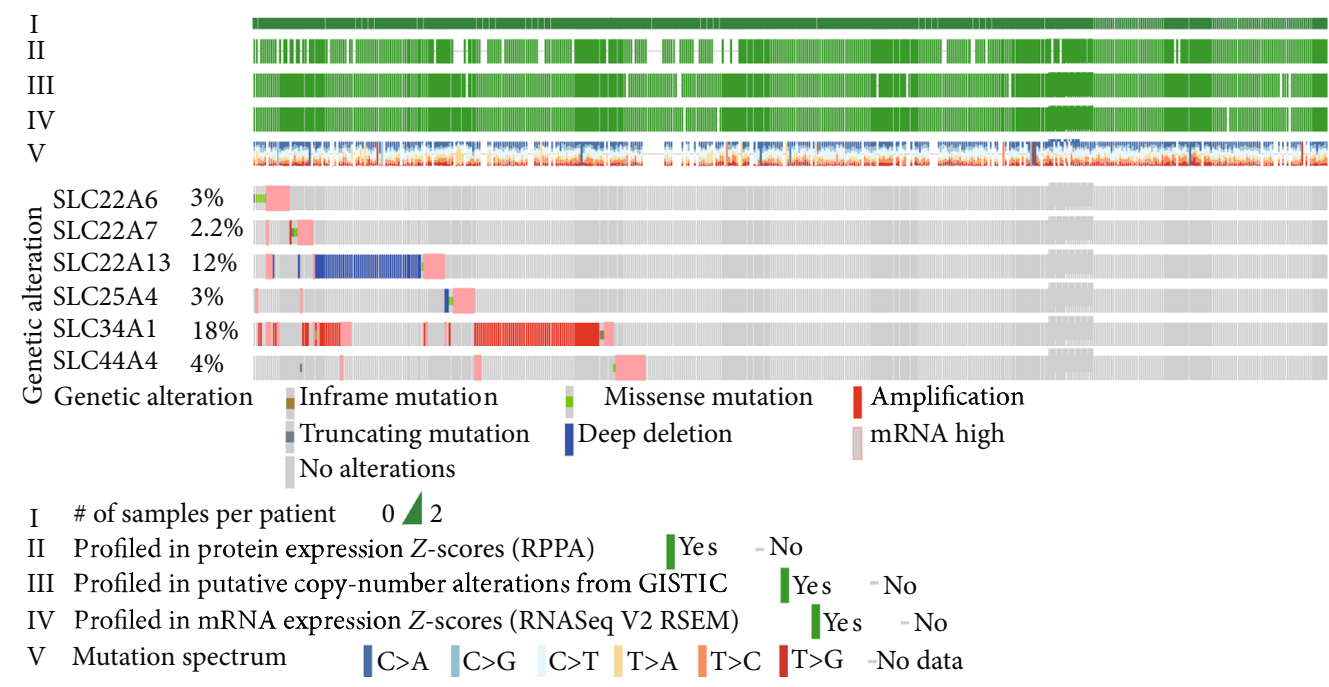

FIgURE 6: The genetic alteration analysis and the correlation of the SLC family genes in ccRCC (cBioPortal).

3.10. The Prognostic Value of the SLC Family Genes Was Reevaluated by the ICGC Dataset. We used the ICGC dataset to verify the prognostic value of the SLC family genes in ccRCC again. The cohort study included 492 patients, including 168 women and 324 men. As shown in Figure 10, the expression levels of SLC22A6, SLC22A7, SLC22A13, SLC25A4, SLC34A1, and SLC44A4 in ccRCC were significantly correlated with the OS, and the difference was statistically significant $(P<0.01)$. High SLC22A6, SLC22A7, SLC22A13, SLC25A4, SLC34A1, and SLC44A4 expression predicted improved OS. The analysis results were the same as before and confirmed that our previous analysis results were reliable.

\section{Discussion}

Metabolic disorders are hallmarks of cancer and offer the potential for cancer diagnosis, prognosis, and treatment [27-30]. Tumors readjust their metabolism to produce enough energy and substances for the proliferation of malignant cells. Recent studies have demonstrated that the pathological accumulation of metabolic intermediates such as fumarate and 2-hydroxyglutarate can contribute to tumorigenesis $[31,32]$. Lipids and glycogen are abundant in ccRCC cells [33], suggesting that fatty acid and glucose metabolism changes during the development of ccRCC. Several studies have shown that metabolic changes play a key role in ccRCC progression [34], and other studies have revealed that poor patient survival is associated with the upregulation of pentose phosphate pathway and fatty acid synthesis pathway genes and the downregulation of the tricarboxylic acid (TCA) cycle genes $[34,35]$. As a metabolic disease, many mutated genes, such as VHL, fumarate hydratase (FH), and succinate dehydrogenase $(\mathrm{SDH})$, are involved in cellular respiration and energy metabolism [7]. HIF-1-induced genetic reprogramming promotes a classic Warburg phenotype in RCC through VHL or mechanisms dependent on metabolic enzymes [7, 36, 37]. Cancer cells predominantly produce energy by lactic acid fermentation, regardless of the oxygen level; this change was known as the Warburg effect or aerobic glycolysis [38, 39]. The loss of VHL leads to a HIF-1dependent reprogramming of energy metabolism that includes elevated glucose uptake, glycolysis, and lactate production accompanied by a reciprocal decrease in respiration under aerobic conditions [36]. Multiple proteomics and metabolomics analyses have shown that compared with normal kidney tissue, the TCA cycle was downregulated between succinate and malate and upregulated between citrate and $\alpha$ ketoglutarate in ccRCC tissues [40-44]. In addition, a combined proteomics and metabolomics study showed increased levels of metabolites in the glutamine and glutathione/oxidized glutathione pathways, including glutamine, glutamate, glutathione, and oxidized glutathione [41].

SLC22A6 was an organic anion/dicarboxylate exchanger. The outwardly directed gradient of $\alpha$-ketoglutarate $(\alpha-K G)$ provides the driving force for the uptake of organic anions against the opposing force of the membrane potential [45]. Endogenous substrates of SLC22A6 include medium-chain fatty acids, $\alpha$-ketoglutarate, citrulline [46], $5^{\prime}$-cyclic adenosine monophosphate (cAMP) and cyclic granulocyte-monocyte progenitor (cGMP) [47], prostaglandins E2 and F2, urate, and acid neurotransmitter metabolites [48]. SLC22A7 mediates cellular efflux of glutamate which may be transstimulated by uptake of some organic anions [49]. In addition to glutamate, SLC22A7 transports the following endogenous compounds into cells: glutarate [50], urate [51], purine and pyrimidine nucleobases, nucleosides and nucleotides, prostaglandin E2, prostaglandin F2, estrone-3-sulfate, allopurinol, and $\alpha$-ketoglutarate $[48,52]$. The endogenous compounds nicotinate, lactate, urate, succinate, and glutathione were identified as substrates of human SLC22A13. Transstimulation of urate uptake by succinate, lactate, and glutathione suggested that human SLC22A13 operates as an anion/anion exchanger [53].

Our study found that SLC22A6, SLC22A7, and SLC22A13 exhibited abnormal changes in various tumors. In ccRCC, the mRNA and protein expression levels of SLC22A6, SLC22A7, 


\begin{tabular}{|c|c|c|c|c|c|c|}
\hline SLC22A6 & 0.14 & 0.33 & 0.01 & 0.22 & -0.09 & \multirow{6}{*}{$\begin{array}{c}\text { Correlation } \\
=-1\end{array}$} \\
\hline \multirow[t]{3}{*}{ - } & SLC22A7 & 0.19 & 0.10 & 0.23 & 0.06 & \\
\hline & ○ & SLC22A13 & 0.03 & 0.37 & -0.03 & \\
\hline & & & SLC25A4 & 0.00 & 0.00 & \\
\hline ○ & O & 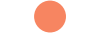 & & SLC34A 1 & 0.04 & \\
\hline & & & & & SLC44A4 & \\
\hline
\end{tabular}

(a)

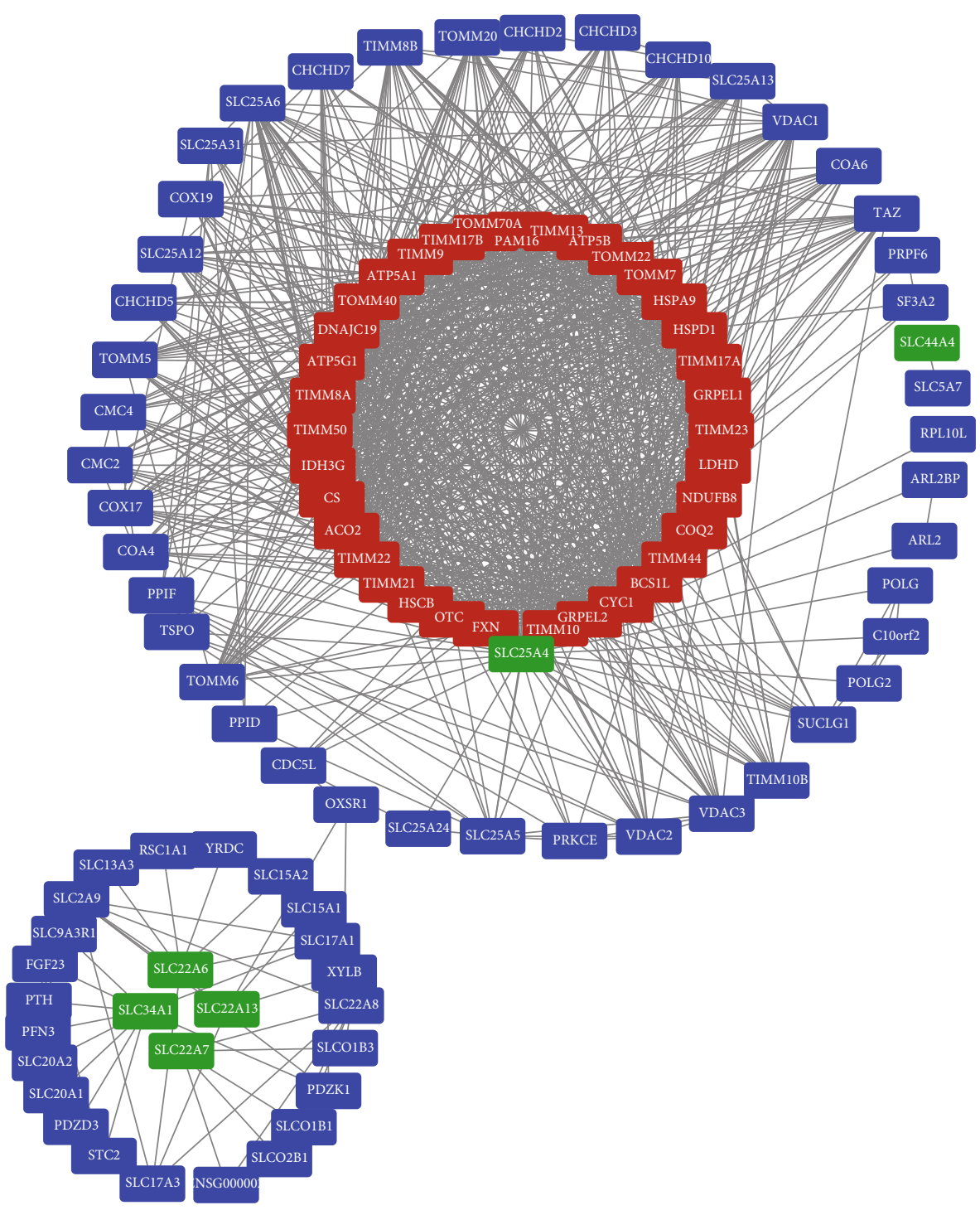

(b)

FIgURe 7: The correlation between different SLC family genes and the PPI network and module analysis. (a) The correlation between different SLC family genes in ccRCC. The size of the circle and the depth of the color represent the strength of the correlation. That is, the larger the circle, the darker the color, and the greater the correlation between genes. (b) The SLC family genes were used to construct the PPI network by STRING (https://string-db.org/), high confidence equals 0.700; the max number of interactors to show were 100). Total genes were mapped by Cytoscape. The MCODE was conducted to select appropriate modules of PPI network. Green indicated the six SLC family genes, red indicated the key modules, and blue indicated other interacting proteins. 

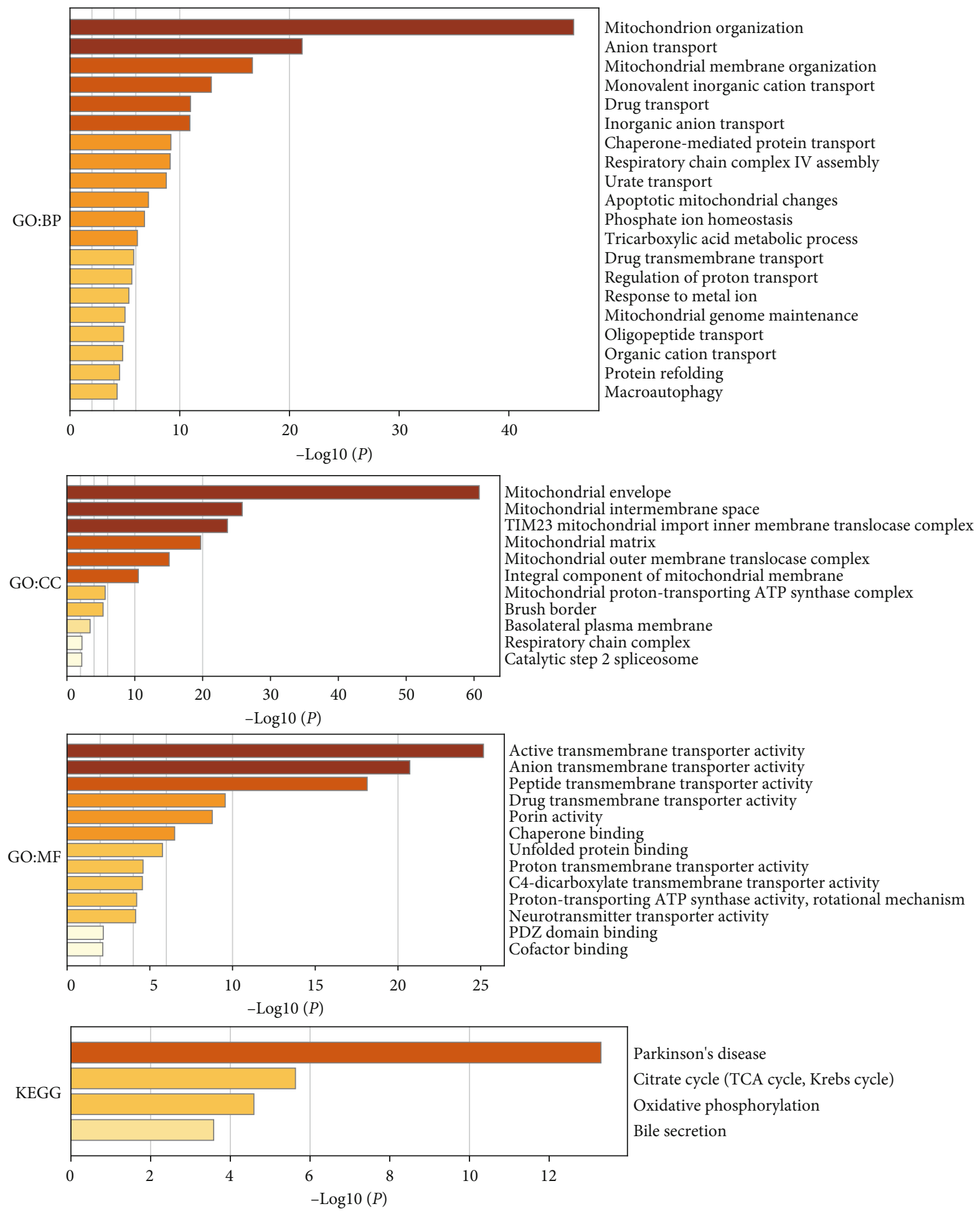

FIGURE 8: Functions of the SLC family genes and genes that interact with the SLC family genes were predicted with functional enrichment analysis and KEGG pathway analysis (Metascape). BP: biological processes; CC: cellular components; MF: molecular functions.

and SLC22A13 were significantly reduced compared to those in normal tissues. Further studies found that the expression levels of SLC22A6 and SLC22A13 were associated with the clinical stage, OS, and DFS. High SLC22A6, SLC22A7, and SLC22A13 expression predicts improved OS and DFS. Other genes in the SLC22 family, such as SLC22A1, SLC22A2, and
SLC22A14, have not changed differentially in ccRCC. Low expression of SLC22A6 led to accumulation of $\alpha$-KG in cells. $\alpha-\mathrm{KG}$ was shown recently to activate $5^{\prime}$ adenosine monophosphate-activated protein kinase (AMPK) to promote anoikis resistance of tumor cells by enhancing its interaction with calcium/calmodulin-dependent protein kinase 


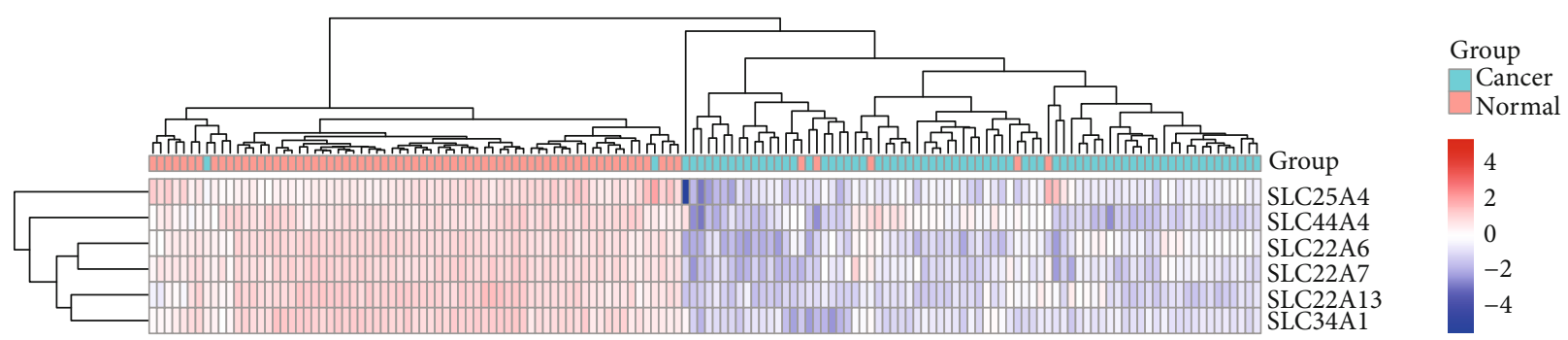

(a)
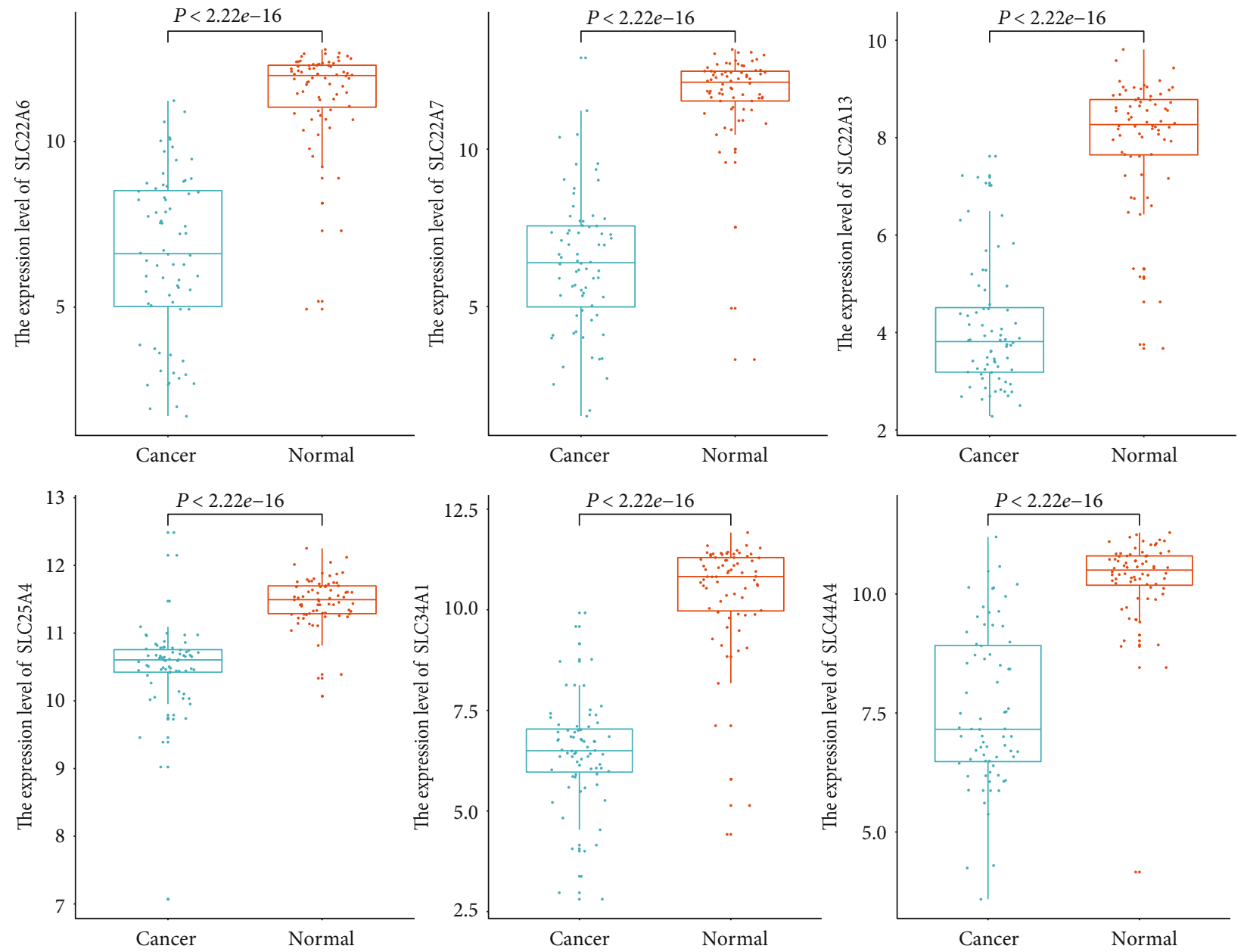

(b)

FIgure 9: The GSE53753 dataset was used to reverify the differential expression level of the SLC family in ccRCC. (a) The heat map showed the expression of the SLC family in normal and cancer tissues. (b) The boxplot showed the expression of the SLC family in normal and cancer tissues. $P<0.01$ meant the difference was statistically significant.

kinase 2 (CamKK2) [54]. In addition, glutamate dehydrogenase 1- (GDH1-) produced $\alpha$-KG directly binds to and activates I-kappaB kinase beta $(\mathrm{IKK} \beta)$ and NF- $\kappa \mathrm{B}$ signaling, which promotes glucose uptake and tumor cell survival by upregulating glucose transporter 1 (GLUT-1) [55]. Low expression of SLC22A7 led to accumulation of glutamate. The upregulation of the glutamine and glutathione/oxidized glutathione pathways correlates with high grade, high stage, and metastasis of ccRCC $[40,41]$. The released glutamine can act as a growth factor and a signal mediator in nonneuronal cancer tissues [56]. Low expression of SLC22A7 led to accumulation of lactate and glutathione. In ccRCC, the increase in GLUT-1 expression correlates with a decrease in the numbers of infiltrating CD8+ T cells [57], suggesting an additional mechanism by which ccRCC might suppress the immune system. This decrease in CD8+ T cells might be the result of increased lactate levels owing to GLUT-1 induction as lactate has been reported to inhibit T-cell activity [58].

In additional, SLC25A4, SLC34A1, and SLC44A4 exhibited abnormal changes in various tumors. In ccRCC, the mRNA expression levels of SLC25A4, SLC34A1, and 

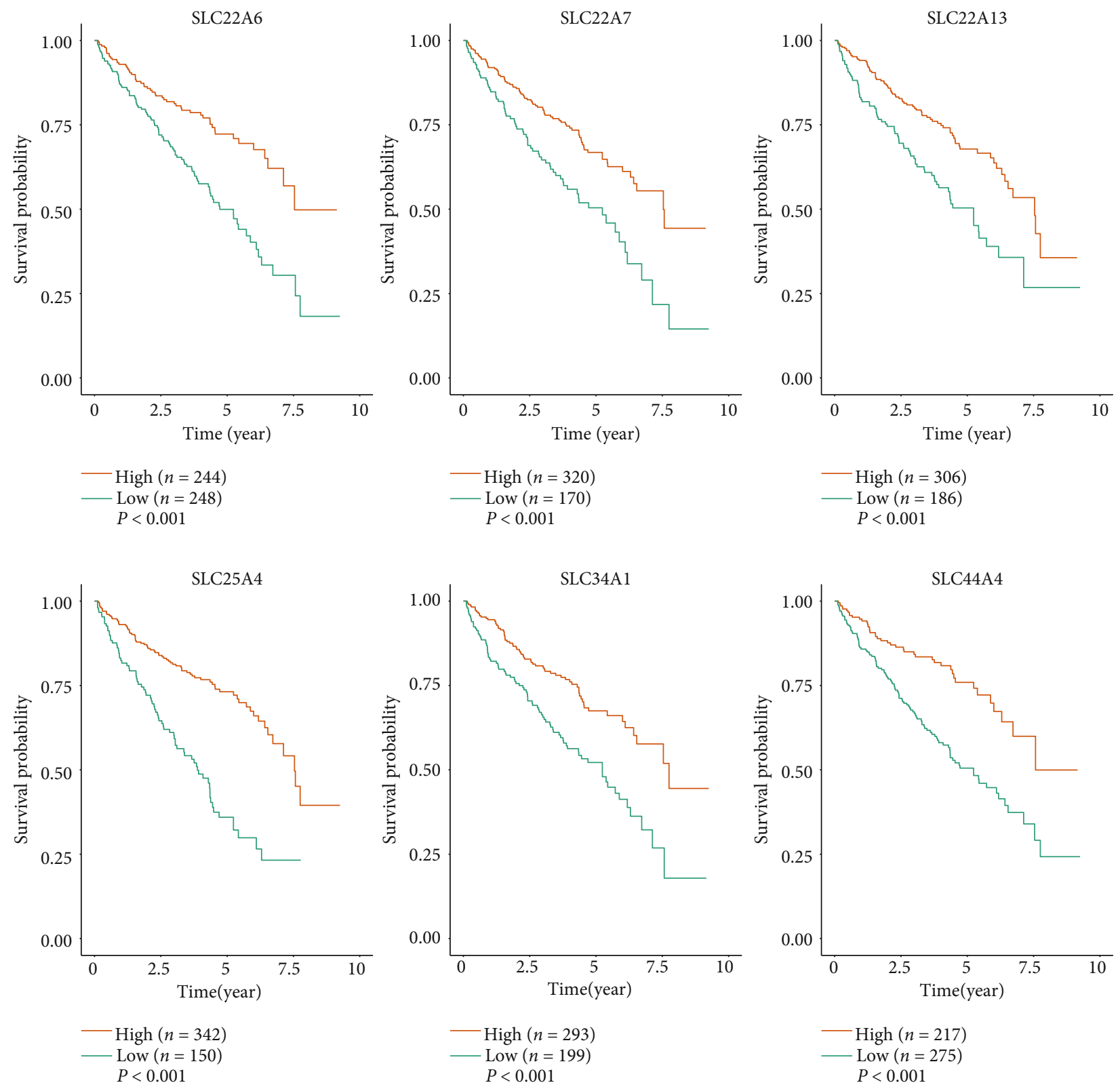

FIGURE 10: The overall survival of the ccRCC patients was reevaluated by the ICGC. $P<0.01$ meant the difference was statistically significant.

SLC44A4 were significantly reduced. Further studies found that the expression levels of SLC25A4, SLC34A1, and SLC44A4 were associated with the clinical stage, OS, and DFS in ccRCC patients. High SLC25A4, SLC34A1, and SLC44A4 expression predicts improved OS and DFS. In ccRCC, the protein expression levels of SLC34A1 were significantly reduced, but the protein expression levels of SLC25A4 and SLC44A4 were not significantly different.

Inorganic phosphate $(\mathrm{Pi})$ is associated with energy metabolism and acts as an integral part of signaling pathways by way of phosphorylation and dephosphorylation reactions of transcription factors and other intermediates of cellular signaling events $[59,60]$. There was a consensus that tumor cells required relatively more phosphate because of their rapid rates of growth $[61,62]$. In skin cancer, Pi was an essen- tial nutrient for cell proliferation and for the promotion of tumorigenesis via the activation of the neuroblastoma RAS viral oncogene homolog (NRas) [63]. Transcellular transport of phosphate is initiated by several apically localized sodiumdependent $\mathrm{Pi}$ cotransporters ( $\mathrm{Na} / \mathrm{Pi}$-cotransporters) that belong to the SLC20 (SLC20A1 and SLC20A2) and SLC34 (SLC34A1, SLC34A2, and SLC34A3) families [64]. All of the members are sodium-driven phosphate cotransporters that promote cell reabsorption of $\mathrm{Pi}$ [10]. In ccRCC, the mRNA and protein expression levels of SLC34A1 were significantly reduced. Theoretically, the decrease of SLC34A1 inhibited reabsorption of $\mathrm{Pi}$, which led to the decrease of $\mathrm{Pi}$ in cells. In fact, the level of $\mathrm{Pi}$ in tumor cells was increased. In ccRCC, there was no differential expression of some transcellular transports responsible for $\mathrm{Pi}$ reabsorption in the 
GEPIA, such as SLC20A1 and SLC20A2, which rescued the reduced $\mathrm{Pi}$ reabsorption caused by SLC34A1. Therefore, we speculated that the functions of SLC34A1 are beyond $\mathrm{Pi}$ transmission. For example, although SLC20A1 was responsible for Pi transport, it was also responsible for activation by $\mathrm{NF}-\kappa \mathrm{B}$, an important protumoral pathway that might be induced by various cytokines [65].

In summary, we systematically analyzed the expression patterns and prognostic values of the SLC family in ccRCC and provided an improved understanding of the heterogeneity and complexity of the molecular biological properties of ccRCC. The results suggest that the downregulation of SLC22A6, SLC22A7, SLC22A13, SLC25A4, SLC34A1, and SLC44A4 in ccRCC may play an important role in the development of ccRCC. The low expression of SLC22A6, SLC22A13, SLC25A4, SLC34A1, and SLC44A4 can also be used as a molecular marker for identifying high-risk subgroups of ccRCC patients. The high expression of SLC22A6, SLC22A7, SLC22A13, SLC25A4, SLC34A1, and SLC44A4 can be used as a potential prognostic indicator for improving survival and prognosis in ccRCC patients.

\section{Conclusions}

Our study found that the expression levels of SLC22A6, SLC22A7, SLC22A13, SLC25A4, SLC34A1, and SLC44A4 were significantly lower in ccRCC tissues. They were significantly associated with clinical stage, OS, and DFS. They may be potential targets for the clinical diagnosis, prognosis, and treatment of ccRCC patients. However, findings should be validated with the clinical sample and functional experiment in the future.

\section{Data Availability}

The datasets used and analysed during the current study are available from the corresponding author on reasonable request.

\section{Conflicts of Interest}

The authors declare that the research was conducted in the absence of any commercial or financial relationships that could be construed as a potential conflict of interest.

\section{Acknowledgments}

The authors acknowledge the entire staff of the Protein Atlas Project for the IHC images and also the entire staff of The Cancer Genome Atlas. The results were partly based upon data generated by the TCGA Research Network (https://www.cancer.gov/tcga). This study was funded by National Natural Science Foundation of China (grant no. 81202016 and grant no. 81572534); China Postdoctoral Science Foundation (grant no. 2016M590638); Medical and Health Technology Development Project of Shandong Province, China (grant no. 2016WS0424); Shandong Medical and Health Science and Technology Development Program (2016WSB01033); and Shandong Key Research and Development Plan (2018GSF118189).

\section{References}

[1] R. L. Siegel, K. D. Miller, and A. Jemal, "Cancer statistics, 2019," CA: a Cancer Journal for Clinicians, vol. 69, no. 1, pp. 7-34, 2019.

[2] A. Lopez-Beltran, M. Scarpelli, R. Montironi, and Z. Kirkali, "2004 WHO classification of the renal tumors of the adults," European Urology, vol. 49, no. 5, pp. 798-805, 2006.

[3] H. Moch, A. L. Cubilla, P. A. Humphrey, V. E. Reuter, and T. M. Ulbright, "The 2016 WHO classification of tumours of the urinary system and male genital organs-part A: renal, penile, and testicular tumours," European Urology, vol. 70, no. 1, pp. 93-105, 2016.

[4] I. J. Frew and H. Moch, "A clearer view of the molecular complexity of clear cell renal cell carcinoma," Annual Review of Pathology, vol. 10, pp. 263-289, 2015.

[5] M. B. Atkins and N. M. Tannir, "Current and emerging therapies for first-line treatment of metastatic clear cell renal cell carcinoma," Cancer Treatment Reviews, vol. 70, pp. 127-137, 2018.

[6] D. J. Sanchez and M. C. Simon, "Genetic and metabolic hallmarks of clear cell renal cell carcinoma," Biochimica Et Biophysica Acta. Reviews on Cancer, vol. 1870, no. 1, pp. 23-31, 2018.

[7] W. M. Linehan, R. Srinivasan, and L. S. Schmidt, "The genetic basis of kidney cancer: a metabolic disease," Nature Reviews. Urology, vol. 7, no. 5, pp. 277-285, 2010.

[8] A. A. Hakimi, C. G. Pham, and J. J. Hsieh, "A clear picture of renal cell carcinoma," Nature Genetics, vol. 45, no. 8, pp. 849-850, 2013.

[9] H. I. Wettersten, O. A. Aboud, Lara PN Jr, and R. H. Weiss, "Metabolic reprogramming in clear cell renal cell carcinoma," Nature Reviews. Nephrology, vol. 13, no. 7, pp. 410-419, 2017.

[10] G. Diallinas, "Understanding transporter specificity and the discrete appearance of channel-like gating domains in transporters," Frontiers in Pharmacology, vol. 5, 2014.

[11] C. Colas, P. M. Ung, and A. Schlessinger, "SLC transporters: structure, function, and drug discovery," Medchemcomm, vol. 7, no. 6, pp. 1069-1081, 2016.

[12] T. Amann and C. Hellerbrand, "GLUT1 as a therapeutic target in hepatocellular carcinoma," Expert Opinion on Therapeutic Targets, vol. 13, no. 12, pp. 1411-1427, 2009.

[13] L. A. Timmerman, T. Holton, M. Yuneva et al., "Glutamine sensitivity analysis identifies the $\mathrm{xCT}$ antiporter as a common triple-negative breast tumor therapeutic target," Cancer Cell, vol. 24, no. 4, pp. 450-465, 2013.

[14] E. Babu, Y. D. Bhutia, S. Ramachandran et al., "Deletion of the amino acid transporter Slc6a14 suppresses tumour growth in spontaneous mouse models of breast cancer," The Biochemical Journal, vol. 469, no. 1, pp. 17-23, 2015.

[15] Q. Wang and J. Holst, "L-type amino acid transport and cancer: targeting the mTORC1 pathway to inhibit neoplasia," American Journal of Cancer Research, vol. 5, no. 4, pp. 12811294, 2015.

[16] P. Nicklin, P. Bergman, B. Zhang et al., "Bidirectional transport of amino acids regulates mTOR and autophagy," Cell, vol. 136, no. 3, pp. 521-534, 2009.

[17] Y. J. Jeon, S. Khelifa, B. Ratnikov et al., "Regulation of glutamine carrier proteins by RNF5 determines breast cancer response to ER stress-inducing chemotherapies," Cancer Cell, vol. 27, no. 3, pp. 354-369, 2015. 
[18] K. Golovine, P. Makhov, R. G. Uzzo, T. Shaw, D. Kunkle, and V. M. Kolenko, "Overexpression of the zinc uptake transporter hZIP1 inhibits nuclear factor-kappaB and reduces the malignant potential of prostate cancer cells in vitro and in vivo," Clinical Cancer Research, vol. 14, no. 17, pp. 5376-5384, 2008.

[19] W. Fangning, M. Chunguang, Z. Hailiang et al., "Identification and validation of soluble carrier family expression signature for predicting poor outcome of renal cell carcinoma," Journal of Cancer, vol. 8, no. 11, pp. 2010-2017, 2017.

[20] Z. Tang, C. Li, B. Kang, G. Gao, C. Li, and Z. Zhang, "GEPIA: a web server for cancer and normal gene expression profiling and interactive analyses," Nucleic Acids Research, vol. 45, no. W1, pp. W98-w102, 2017.

[21] J. Gao, B. A. Aksoy, U. Dogrusoz et al., "Integrative analysis of complex cancer genomics and clinical profiles using the cBioPortal," Science Signaling, vol. 6, no. 269, p. pl1, 2013.

[22] E. Cerami, J. Gao, U. Dogrusoz et al., "The cBio cancer genomics portal: an open platform for exploring multidimensional cancer genomics data," Cancer Discovery, vol. 2, no. 5, pp. 401404, 2012.

[23] D. Szklarczyk, A. Franceschini, M. Kuhn et al., "The STRING database in 2011: functional interaction networks of proteins, globally integrated and scored," Nucleic Acids Research, vol. 39, no. Database issue, pp. D561-D568, 2011.

[24] M. E. Smoot, K. Ono, J. Ruscheinski, P. L. Wang, and T. Ideker, "Cytoscape 2.8: new features for data integration and network visualization," Bioinformatics, vol. 27, no. 3, pp. 431-432, 2011.

[25] G. D. Bader and C. W. Hogue, "An automated method for finding molecular complexes in large protein interaction networks," BMC Bioinformatics, vol. 4, no. 1, p. 2, 2003.

[26] Y. Zhou, B. Zhou, L. Pache et al., "Metascape provides a biologist-oriented resource for the analysis of systems-level datasets," Nature Communications, vol. 10, no. 1, p. 1523, 2019.

[27] R. J. DeBerardinis, J. J. Lum, G. Hatzivassiliou, and C. B. Thompson, "The biology of cancer: metabolic reprogramming fuels cell growth and proliferation," Cell Metabolism, vol. 7, no. 1, pp. 11-20, 2008.

[28] D. Hanahan and R. A. Weinberg, "Hallmarks of cancer: the next generation," Cell, vol. 144, no. 5, pp. 646-674, 2011.

[29] P. P. Hsu and D. M. Sabatini, "Cancer cell metabolism: Warburg and beyond," Cell, vol. 134, no. 5, pp. 703-707, 2008.

[30] M. G. V. Heiden, L. C. Cantley, and C. B. Thompson, "Understanding the Warburg effect: the metabolic requirements of cell proliferation," Science, vol. 324, no. 5930, pp. 1029-1033, 2009.

[31] W. G. Kaelin Jr. and S. L. McKnight, "Influence of metabolism on epigenetics and disease," Cell, vol. 153, no. 1, pp. 56-69, 2013.

[32] N. Raimundo, B. E. Baysal, and G. S. Shadel, "Revisiting the TCA cycle: signaling to tumor formation," Trends in Molecular Medicine, vol. 17, no. 11, pp. 641-649, 2011.

[33] R. L. Gebhard, R. V. Clayman, W. F. Prigge et al., “Abnormal cholesterol metabolism in renal clear cell carcinoma," Journal of Lipid Research, vol. 28, no. 10, pp. 1177-1184, 1987.

[34] The Cancer Genome Atlas Research Network, "Comprehensive molecular characterization of clear cell renal cell carcinoma," Nature, vol. 499, no. 7456, pp. 43-49, 2013.

[35] A. A. Hakimi, H. Furberg, E. C. Zabor et al., "An epidemiologic and genomic investigation into the obesity paradox in renal cell carcinoma," Journal of the National Cancer Institute, vol. 105, no. 24, pp. 1862-1870, 2013.

[36] G. L. Semenza, "HIF-1 mediates the Warburg effect in clear cell renal carcinoma," Journal of Bioenergetics and Biomembranes, vol. 39, no. 3, pp. 231-234, 2007.

[37] S. Sudarshan, C. Sourbier, H. S. Kong et al., "Fumarate hydratase deficiency in renal cancer induces glycolytic addiction and hypoxia-inducible transcription factor 1alpha stabilization by glucose-dependent generation of reactive oxygen species," Molecular and Cellular Biology, vol. 29, no. 15, pp. 40804090, 2009.

[38] O. Warburg, F. Wind, and E. Negelein, "The metabolism of tumors in the body," The Journal of General Physiology, vol. 8, no. 6, pp. 519-530, 1927.

[39] P. S. Ward and C. B. Thompson, "Metabolic reprogramming: a cancer hallmark even Warburg did not anticipate," Cancer Cell, vol. 21, no. 3, pp. 297-308, 2012.

[40] A. A. Hakimi, E. Reznik, C. H. Lee et al., "An integrated metabolic atlas of clear cell renal cell carcinoma," Cancer Cell, vol. 29, no. 1, pp. 104-116, 2016.

[41] H. I. Wettersten, A. A. Hakimi, D. Morin et al., "Grade-dependent metabolic reprogramming in kidney cancer revealed by combined proteomics and metabolomics analysis," Cancer Research, vol. 75, no. 12, pp. 2541-2552, 2015.

[42] B. Perroud, T. Ishimaru, A. D. Borowsky, and R. H. Weiss, "Grade-dependent proteomics characterization of kidney cancer," Molecular \& cellular proteomics : MCP, vol. 8, no. 5, pp. 971-985, 2009.

[43] B. Perroud, J. Lee, N. Valkova et al., "Pathway analysis of kidney cancer using proteomics and metabolic profiling," Molecular Cancer, vol. 5, no. 1, pp. 64-64, 2006.

[44] G. Catchpole, A. Platzer, C. Weikert et al., "Metabolic profiling reveals key metabolic features of renal cell carcinoma," Journal of Cellular and Molecular Medicine, vol. 15, no. 1, pp. 109-118, 2011.

[45] B. C. Burckhardt and G. Burckhardt, "Transport of organic anions across the basolateral membrane of proximal tubule cells," Reviews of Physiology, Biochemistry and Pharmacology, vol. 146, pp. 95-158, 2003.

[46] M. Nakakariya, Y. Shima, Y. Shirasaka, K. Mitsuoka, T. Nakanishi, and I. Tamai, "Organic anion transporter OAT1 is involved in renal handling of citrulline," Renal Physiology, vol. 297, no. 1, pp. F71-F79, 2009.

[47] C. D. Cropp, T. Komori, J. E. Shima et al., "Organic anion transporter 2 (SLC22A7) is a facilitative transporter of cGMP," Molecular Pharmacology, vol. 73, no. 4, pp. 11511158, 2008.

[48] A. N. Rizwan and G. Burckhardt, "Organic anion transporters of the SLC22 family: biopharmaceutical, physiological, and pathological roles," Pharmaceutical Research, vol. 24, no. 3, pp. 450-470, 2007.

[49] C. Fork, T. Bauer, S. Golz et al., "OAT2 catalyses efflux of glutamate and uptake of orotic acid," The Biochemical Journal, vol. 436, no. 2, pp. 305-312, 2011.

[50] Y. Kobayashi, N. Ohshiro, R. Sakai, M. Ohbayashi, N. Kohyama, and T. Yamamoto, "Transport mechanism and substrate specificity of human organic anion transporter 2 (hOat2 [SLC22A7])," The Journal of Pharmacy and Pharmacology, vol. 57, no. 5, pp. 573-578, 2005.

[51] M. Sato, H. Mamada, N. Anzai, Y. Shirasaka, T. Nakanishi, and I. Tamai, "Renal secretion of uric acid by organic anion 
transporter 2 (OAT2/SLC22A7) in human," Biological \& Pharmaceutical Bulletin, vol. 33, no. 3, pp. 498-503, 2010.

[52] C. Srimaroeng, J. L. Perry, and J. B. Pritchard, "Physiology, structure, and regulation of the cloned organic anion transporters," Xenobiotica; the fate of foreign compounds in biological systems, vol. 38, no. 7-8, pp. 889-935, 2008.

[53] H. Koepsell, "The SLC22 family with transporters of organic cations, anions and zwitterions," Molecular Aspects of Medicine, vol. 34, no. 2-3, pp. 413-435, 2013.

[54] L. Jin, J. Chun, C. Pan et al., "The PLAG1-GDH1 Axis Promotes Anoikis Resistance and Tumor Metastasis through CamKK2-AMPK Signaling in LKB1-Deficient Lung Cancer," Molecular cell, vol. 69, no. 1, pp. 87-99.e7, 2018.

[55] X. Wang, R. Liu, X. Qu et al., " $\alpha$-Ketoglutarate-activated NF$\kappa \mathrm{B}$ signaling promotes compensatory glucose uptake and brain tumor development," Molecular cell, vol. 76, no. 1, article S1097276519305374, pp. 148-162.e7, 2019.

[56] A. Stepulak, R. Rola, K. Polberg, and C. Ikonomidou, "Glutamate and its receptors in cancer," Journal of neural transmission (Vienna, Austria: 1996), vol. 121, no. 8, pp. 933-944, 2014.

[57] K. Singer, M. Kastenberger, E. Gottfried et al., "Warburg phenotype in renal cell carcinoma: high expression of glucosetransporter 1 (GLUT-1) correlates with low CD8(+) T-cell infiltration in the tumor," International Journal of Cancer, vol. 128, no. 9, pp. 2085-2095, 2011.

[58] K. Fischer, P. Hoffmann, S. Voelkl et al., "Inhibitory effect of tumor cell-derived lactic acid on human T cells," Blood, vol. 109, no. 9, pp. 3812-3819, 2007.

[59] L. Sapio and S. Naviglio, "Inorganic phosphate in the development and treatment of cancer: a Janus Bifrons?," World journal of clinical oncology, vol. 6, no. 6, pp. 198-201, 2015.

[60] H. Jin, C. X. Xu, H. T. Lim et al., "High dietary inorganic phosphate increases lung tumorigenesis and alters Akt signaling," American Journal of Respiratory and Critical Care Medicine, vol. 179, no. 1, pp. 59-68, 2009.

[61] J. J. Elser and A. Hamilton, "Stoichiometry and the new biology: the future is now," PLoS Biology, vol. 5, no. 7, pp. e181e181, 2007.

[62] I. Kareva, "Biological stoichiometry in tumor micro-environments,” PLoS One, vol. 8, no. 1, pp. e51844-e51844, 2013.

[63] C. E. Camalier, M. R. Young, G. Bobe, C. M. Perella, N. H. Colburn, and G. R. Beck, "Elevated phosphate activates N-ras and promotes cell transformation and skin tumorigenesis," Cancer prevention research (Philadelphia, Pa.), vol. 3, no. 3, pp. 359$370,2010$.

[64] H. Murer and J. Biber, "Phosphate transport in the kidney," Journal of Nephrology, vol. 23, Supplement 16, pp. S145S151, 2010.

[65] C. Salaün, C. Leroy, A. Rousseau, V. Boitez, L. Beck, and G. Friedlander, "Identification of a novel transportindependent function of PiT1/SLC20A1 in the regulation of TNF-induced apoptosis," The Journal of Biological Chemistry, vol. 285, no. 45, pp. 34408-34418, 2010. 